\title{
An Integrated Model to Characterize Comprehensive Stiffness of Angular Contact Ball Bearings
}

\author{
Chunli Lei ${ }^{1},{ }^{1}$ Fuhong Li, ${ }^{2}$ Baoru Gong, ${ }^{1}$ and Xibin Jia ${ }^{1}$ \\ ${ }^{1}$ Key Laboratory of Digital Manufacturing Technology and Application, Lanzhou University of Technology, \\ Lanzhou 730050, China \\ ${ }^{2}$ New Oriental Education and Technology Group, Lanzhou 730030, China
}

Correspondence should be addressed to Chunli Lei; lclyq2004@163.com

Received 2 September 2019; Revised 24 February 2020; Accepted 2 March 2020; Published 7 April 2020

Academic Editor: Gregory Chagnon

Copyright (C) 2020 Chunli Lei et al. This is an open access article distributed under the Creative Commons Attribution License, which permits unrestricted use, distribution, and reproduction in any medium, provided the original work is properly cited.

\begin{abstract}
The bearing dynamic behaviors will be complicated due to the changes in the geometric sizes and relative positions of the bearing components at high speed. In this paper, based on the Hertz contact theory, elastohydrodynamic lubrication (EHL) model, and Jones' bearing theory, the comprehensive stiffness model of the angular contact ball bearing is proposed in consideration of the effects of elastic deformation, centrifugal deformation, thermal deformation, and the ball spinning motion. The influences of these factors on bearing dynamic stiffness are investigated in detail. The calculation results show that the centrifugal deformation and thermal deformation increase with the increase in rotation speed. When the centrifugal deformation and thermal deformation are considered, the bearing radial contact stiffness increases as the speed increases, whereas the axial contact stiffness and the angular contact stiffness decrease. When the deformations and the EHL are all considered, the comprehensive bearing stiffness decreases with the increasing speed. It is also found that the spinning motion of the ball causes the comprehensive bearing stiffness to increase.
\end{abstract}

\section{Introduction}

Development of a spindle-bearing system can significantly improve the efficiency and accuracy of machine tools. The angular contact ball bearing is the kernel of high-speed motorized spindles, and the $\mathrm{dN}$ value is more than $0.6 \times 10^{6} \mathrm{~mm} \cdot(\mathrm{r} / \mathrm{min})$. Its dynamic stiffness directly affects the machining accuracy and dynamic characteristics of the spindle [1-5]. The critical speed, vibration characteristics, and reliability of the bearing-rotor system are dependent on the bearing stiffness [6-8]. In this sense, it is necessary to analyze the internal dynamic state of the spindle bearing at high speed and to investigate the influencing factors and change laws of bearing dynamic stiffness. Consequently, establishment of a perfect bearing dynamic stiffness model is of great significance to optimize the structural parameters of the bearing, improve the bearing rigidity at high speed, and improve the running accuracy of the spindle.
Many scholars have carried out a great deal of research studies on the stiffness characteristics of rolling bearings. Jones [9] established the bearing dynamics model considering the effect of centrifugal force and gyroscopic torque. Harris et al. $[10,11]$ considered the influence of the elastohydrodynamic lubrication on the bearing dynamic characteristics in his model. Later, researchers further studied the dynamic characteristics of high-speed rolling bearings based on Jones' model and Harris' theory. Gupta [12] constructed a bearing dynamic analysis model taking into account the ball movement, stress state, and the interaction between the various components. Lin et al. [13] presented an integrated model for studying various thermo-mechanical-dynamic spindle behaviors at high speed and investigated the bearing preload effects on bearing stiffness. Cao et al. [14] compared the influences of the speed on the bearing stiffness under different preload mechanisms. Several researchers put forward different stiffness matrixes of angular contact ball bearings, respectively, $[15,16]$. Noel et al. [17] presented a 
new method to calculate the stiffness matrix based on the dynamic model of angular contact ball bearings. A bearing model based on Jones' bearing model was established by Cao and Altintas [18]. Fang et al. [19] deduced the analytic expression of the stiffness of the angular contact ball bearing according to the Hertz elastic contact theory. Hagiu and Gafitanu [20] presented a dynamic model, and both film squeeze effects and elastic deformation of the Hertzian contact were considered. An EHL model to study the effect of spin motion on load distribution and oil film shape was carried out by Guo et al. [21]. Guo and Parker [22] constructed a finite element/contact mechanics model to calculate stiffness matrix of the rolling element bearing. Xiong et al. [23] established a bearing quasi-static modified model considering the effects of ring deformation and EHL. Liu et al. [24] developed a new analytic method considering the axial preload and contact angle for solving the internal load distribution and stiffness of the bearing, and the changes in the contact angle, internal load distribution, and axial stiffness caused by the combined loads were studied.

When the spindle rotates at high speed, the heat generated by friction of the bearing and power loss of the motor can cause expansion of the components of the bearing. The centrifugal force induces the deformation of the parts, and the ball spinning also influences the feature of the lubricating oil film. The traditional quasi-static method cannot reflect the changes of the internal geometric relationship of the bearing under working conditions completely, and the existing calculating models cannot fully include these factors. In this paper, a new comprehensive bearing stiffness model is proposed, which includes the contact stiffness with the consideration of the centrifugal deformation and the thermal deformation and the film stiffness with the consideration of spin motion. An efficient solution is obtained based on the Hertz contact theory for the calculation of forces, Reynolds equation approach with the spinning speed for the EHL problem, and Jones' bearing model for the dynamic parameter. The presented model is verified by the previous work, and the influences of rotational speed, centrifugal deformation, thermal deformation, and elastohydrodynamics on dynamic bearing stiffness are investigated. The proposed dynamic model of the angular contact ball bearing provides more accurate description of dynamic stiffness change laws under high-speed operating conditions. The calculation results are more close to the actual working state of the ball bearing at high speed, which provides a useful reference for the dynamic performance analysis of the rotor system supported by the rolling bearing.

\section{Calculation of Film Stiffness considering Spinning}

The centrifugal force of the ball will increase when the angular contact ball bearing runs at high speed. Subsequently, the spinning motion will occur on the raceway, which not only causes the frictional heating but also changes the thickness of the oil film.

2.1. Calculation of Spin Angular Velocity. For high-speed angular contact ball bearings, gyroscopic moment and spinning motion will inevitably induct large sliding between the balls and raceways and increase the bearing friction moment, which are the main reasons for the bearing heat generation. Therefore, accurately analyzing the spin angular velocity between ball and raceways is benefit for assessment of the friction heat generation rate.

The attitude angle of the rolling element of the ball bearing is one of the important parameters for calculating the spin angular velocity. The attitude angle not only reflects the operating status of the roller but also affects the calculation of various angular velocities of the bearing and the relative sliding movement on the contact area. In this paper, the attitude angle can be expressed as follows [25]:

$$
\begin{aligned}
\beta & =a c \tan \left[\frac{\left(M_{s i} / M_{s o}\right)\left(\left(\left(1+\gamma_{o}\right) /\left(1-\gamma_{i}\right)\right)+1\right) \sin \alpha_{i}+2 \sin \alpha_{o}}{\left(M_{s i} / M_{s o}\right)\left(\left(\left(1+\gamma_{o}\right) /\left(1-\gamma_{i}\right)\right)+1\right) \cos \alpha_{i}+2\left(\cos \alpha_{o}+\gamma^{\prime}\right)+A}\right], \\
A & =\frac{M_{s i}}{M_{s o}} \gamma^{\prime}\left[\cos \left(\alpha_{i}-\alpha_{o}\right)-\frac{1+\gamma_{o}}{1-\gamma_{i}}\right], \\
M_{s i} & =\frac{3 K_{s i} Q_{i j} \mu_{i} \varsigma_{i}}{8}, \\
M_{s o} & =\frac{3 K_{s o} Q_{o j} \mu_{o} \varsigma_{o}}{8},
\end{aligned}
$$

where $\alpha_{i}$ and $\alpha_{o}$ are the contact angles of inner and outer raceways, respectively, $\gamma_{i}=\left(D / d_{m}\right) \cos \alpha_{i}, \gamma_{o}=$ $\left(D / d_{m}\right) \cos \alpha_{o}, \gamma^{\prime}=D / d_{m}, D$ is the ball diameter, $d_{m}$ is the pitch diameter, $M_{s i}$ and $M_{s o}$ is the spinning friction moment of the inner and outer ring, $Q_{i j}$ and $Q_{o j}$ are the contact loads at the inner and outer ring, and $K_{s i}$ and $K_{s o}$ are the friction coefficient of the inner and outer ring, respectively. 
There is a rotation about a normal to the contact area; in other words, a spinning of the outer raceway relative to the ball, when the outer ring is assumed to be fixed; spinning angular speed $\omega_{s i}$ and $\omega_{s o}$ can be described as follows [26]:

$$
\begin{aligned}
& \omega_{s i}=\left(\omega_{i}-\omega_{m}\right) \sin \alpha_{i}-\omega_{r} \sin \left(\beta-\alpha_{i}\right), \\
& \omega_{s o}=\omega_{m} \sin \alpha_{o}-\omega_{r} \sin \left(\alpha_{o}-\beta\right),
\end{aligned}
$$

where $\omega_{i}$ is the inner-ring angular rotation, $\omega_{m}$ is the orbital speed of the ball, and $\omega_{r}$ is the speed of the ball about its own axis:

$$
\begin{aligned}
& \omega_{m}=\frac{\omega_{i}\left(1-\gamma_{i}\right) \cos \left(\beta-\alpha_{o}\right)}{\left(1+\gamma_{o}\right) \cos \left(\beta-\alpha_{i}\right)+\left(1-\gamma_{i}\right) \cos \left(\beta-\alpha_{o}\right)}, \\
& \omega_{r}=\frac{\omega_{i}\left(1-\gamma_{i}\right)\left(1+\gamma_{o}\right)}{\gamma^{\prime}\left[\left(1+\gamma_{o}\right) \cos \left(\beta-\alpha_{i}\right)+\left(1-\gamma_{i}\right) \cos \left(\beta-\alpha_{o}\right)\right]} .
\end{aligned}
$$

2.2. Governing Equations for Lubrication. It is assumed that the lubrication contact between the ball and the raceway is equivalent to the contact between the infinite plane and the ellipsoid, and the plane is rotated at the spin angular velocity $\omega_{s}$, and the ellipsoid rolls at the average speed $u_{b}$ of the rolling element and the raceway [26]. The speed vector for a single ball in a bearing is shown in Figure 1.

It can be seen that the expressions of entrainment speed along $x$-direction and $y$-direction are as follows:

$$
\begin{aligned}
& u_{R}(x, y)=\frac{\omega_{s} y}{2}+u_{b}, \\
& v_{R}(x, y)=\frac{\omega_{s} x}{2} .
\end{aligned}
$$

The Reynolds equation for a steady-state point contact lubricated with a generalized Newtonian lubricant under the spinning condition is given by $\mathrm{Wu}[26]$ :

$$
\frac{\partial}{\partial x}\left(\frac{\rho h^{3}}{\eta} \frac{\partial p}{\partial x}\right)+\frac{\partial}{\partial y}\left(\frac{\rho h^{3}}{\eta} \frac{\partial p}{\partial y}\right)=12\left(\frac{\partial\left(u_{R} \rho h\right)}{\partial x}\right)+12\left(\frac{\partial\left(v_{R} \rho h\right)}{\partial y}\right),
$$

where $p$ is the pressure, $h$ is the lubricant film thickness, $\rho$ is the density of lubricant, and $\eta$ is the lubricant viscosity.

The lubricant film thickness equation at the contact area can be written as

$$
h(x, y)=h_{0}+\frac{x^{2}}{2 R_{x}}+\frac{y^{2}}{2 R_{y}}+\frac{2}{\pi E^{\prime}} \iint_{\Omega} \frac{p(\xi, \lambda)}{\sqrt{(x-\xi)^{2}+(y-\lambda)^{2}}} \mathrm{~d} \xi \mathrm{d} \lambda
$$

where $h_{0}$ is the central film thickness between two solid bodies, $R_{x}$ and $R_{y}$ are equivalent curvature radius of the ball along $x$-direction and $y$-direction, and $E^{\prime}$ is the equivalent elastic modulus:

$$
E^{\prime}=\left(\frac{1-v_{1}^{2}}{2 E_{1}}+\frac{1-v_{2}^{2}}{2 E_{2}}\right)^{-1}
$$

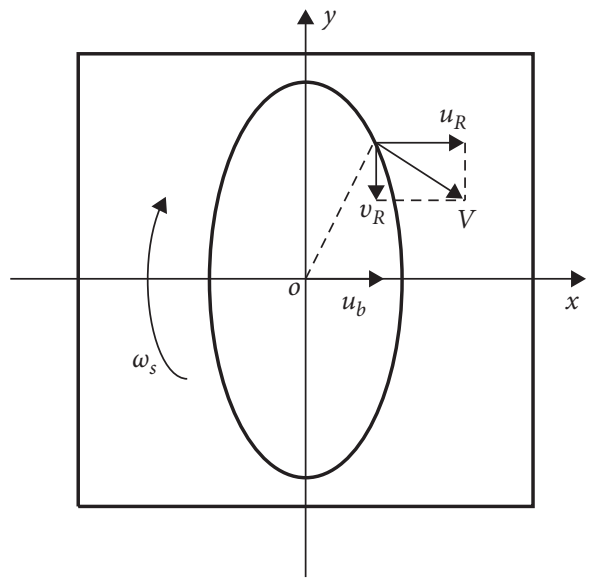

FIgURE 1: Ball speed vector in spinning motion.

where $E_{1}$ and $E_{2}$ are the elasticity modulus of the ring and ball and $v_{1}$ and $v_{2}$ are Poisson's ratio of the ring and ball, respectively.

The pressure distribution should be balanced with the applied load through the following load integration over the computation domain:

$$
w=\int_{x_{0}}^{x_{e}} \int_{y_{0}}^{y_{e}} p \mathrm{~d} x \mathrm{~d} y .
$$

The lubricant viscosity $\eta$ is calculated by using the Roelands equation [27]:

$$
\begin{aligned}
& \eta=\eta_{0} \exp \left\{\left(\ln \eta_{0}+9.67\right)\left[-1+\left(1+5.1 \times 10^{-9} p\right)^{Z}\right]\right\}, \\
& Z=\frac{\alpha}{5.1 \times 10^{-9}\left(\ln \eta_{0}+9.67\right)}
\end{aligned}
$$

in which $\eta_{0}$ denotes the initial dynamic viscosity, $\alpha$ is the viscosity-pressure coefficient, and $Z$ is the coefficient.

The lubricant density $\rho$ is calculated by using the Dowson-Higginson equation [28]:

$$
\rho=\rho_{0}\left(1+\frac{0.6 \times 10^{-9} p}{1+1.7 \times 10^{-9} p}\right)
$$

where $\rho_{0}$ is the ambient density.

Equations (6)-(13) formulate the lubrication problem with spinning effect. Solving this set of equations can provide the basic information for pressuring distribution, oil film thickness, and so on. The dimensionless minimum film thickness by numerical calculation can be obtained. Its dimensionless form can be written as follows [26]:

$$
\begin{aligned}
H_{\min }= & 8.9 \times 10^{-3} U^{0.71964}[1+1.04317 \exp (-664.016 W)] \\
& \times G^{0.80053} k^{1.61623}[1+21.4595 \exp (-0.29996 k)] \\
& \times\left(1-1.3101 \times 10^{10} \Omega_{s}^{1.02415}\right)
\end{aligned}
$$

where $k$ is the ellipticity, $\Omega_{s}$ is the spinning angular velocity parameter, $U$ is the speed parameter, $W$ is the load 
parameter, and $G$ is the material parameter. They can be expressed as follows:

$$
\begin{gathered}
\Omega_{s}=\frac{\omega_{s} \eta_{0}}{E^{\prime}}, \\
U=\frac{u_{b} \eta_{0}}{E^{\prime} R_{x}}, \\
W=\frac{w}{E^{\prime} R_{x}^{2}}, \\
G=\alpha E^{\prime} .
\end{gathered}
$$

The minimum film thickness can be expressed as follows:

$$
h_{\min }=R_{x} H_{\min } \text {. }
$$

2.3. Film Stiffness Model of the Bearing. Equations (14) and (19) are then used to determine the relationship between $W$ and $h_{\text {min }}$ :

$$
\begin{aligned}
W= & (-0.001506) \\
& \ln \left[0.1077 \times 10^{3} h_{\min } \times\left(G^{0.80053} R_{x} U^{0.71964} k^{1.61623}\right.\right. \\
& \cdot(1+21.4595 \exp (-0.29996 k)) \\
& \left.\left.\times\left(1-1.3101 \times 10^{10} \Omega^{1.02415}\right)\right)-0.9586\right] .
\end{aligned}
$$

According to [26], the film stiffness can be expressed as in the following equation:

$K_{\text {oil }}=\frac{\mathrm{d} w}{\mathrm{~d} h_{\min }}=\frac{0.16155 E^{\prime} R_{s}}{\left\{G^{0.80053} U^{0.71964 k} k^{1.61623}(1+21.4595 \exp (-0.29996 k)) \times\left(1-1.3101 \times 10^{10} \Omega_{s}^{1.02415}\right) \times\left[0.1077 \times 10^{3} h_{\min } /\left(G^{0}\right.\right.\right.}$

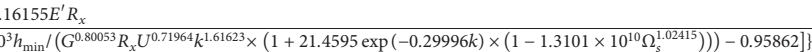

\section{Quasi-Static Modified Model of Angular Contact Ball Bearing}

3.1. Inner-Ring Deformation due to Centrifugal Effect. When the motorized spindle rotates at high speed, the fit between the bearing outer raceway and the housing becomes the interference fit due to the influence of temperature rise. Therefore, centrifugal force is not generated by the out raceway and the housing, whereas the centrifugal force generated by the shaft and the inner raceway will cause the centrifugal deformation of them, respectively. The centrifugal deformation is proportional to the square of the speed. When the speed increases, the centrifugal expansion of the inner raceway is larger than that of the spindle at the contact area and the centrifugal expansion of the shaft cannot change the radial clearance of the bearing [23], so only the inner raceway centrifugal deformation will be taken into consideration in this paper. According to the theory of elastic mechanics, the inner raceway can be simplified as a thin-walled ring, and the calculation formula of the centrifugal expansion of the inner raceway is deduced as follows [29]:

$$
\varepsilon_{c i}=\frac{\rho_{i} \omega_{i}^{2}}{4 E_{i}}\left[\left(3+v_{i}\right) r_{1}^{2}+\left(1-v_{i}\right) r_{2}^{2}\right] r_{2},
$$

where $\rho_{i}$ is the density of the inner raceway, $v_{i}$ is Poisson's ratio of the inner raceway, $E_{i}$ is elasticity modulus of the inner raceway, and $r_{1}$ and $r_{2}$ are the inner and outer radius of the inner raceway, respectively.
3.2. Bearing Deformation due to Thermal Effect. When rotation speed increases, the temperature of the overall motorized spindle increases because of the heat generated by loss of the electrical input power of the motor and the bearing friction moment. Therefore, thermal expansion of the bearing components will be caused, which can be determined according to the material property, geometry size, and the temperature rise of the bearing. The temperature rises are obtained using the model presented in [30].

The ball is assumed to have a uniform temperature and would expand according to the following equation:

$$
\varepsilon_{b}=a_{b} \Delta T_{b} r_{b},
$$

where $a_{b}$ is the thermal expansion coefficient of the ball, $\Delta T_{b}$ is the temperature rise of the ball, and $r_{b}$ is the radius of the ball.

The axial expansion equations of the spindle and the housing can be expressed as

$$
\begin{gathered}
\varepsilon_{s}=\frac{L_{s}}{2} a_{s} \Delta T_{s}, \\
\varepsilon_{h}=\frac{L_{h}}{2} a_{h} \Delta T_{h},
\end{gathered}
$$

where $L_{s}$ and $L_{h}$ are the effective length of spindle and housing, respectively, $a_{s}$ and $a_{h}$ are the thermal expansion coefficient of spindle and housing, and $\Delta T_{s}$ and $\Delta T_{h}$ are the temperature rise of spindle and housing, respectively.

According to the structural characteristics of the motorized spindle, that is, the axial symmetry and uniformity, the radial thermal displacement of the axisymmetric solid 
cylinder can be derived from the stress-strain relationship. Assuming that the radius of the cylinder is $R$, the radial thermal displacement equation at optional position $r$ is expressed as follows [31]:

$$
\varepsilon=a \frac{1+v}{1-v}\left[\frac{1}{r} \int_{0}^{r} \Delta \operatorname{Tr} \mathrm{d} r+\frac{1-2 v}{R^{2}} r \int_{0}^{R} \Delta \operatorname{Tr} \mathrm{d} r\right] .
$$

According to equation (26), radial expansions of the inner raceway-spindle and the outer raceway-housing can be obtained as follows [32]:

$$
\begin{aligned}
& \varepsilon_{i r}=\frac{a_{i}}{3}\left(\Delta T_{s}+\Delta T_{i}\right)\left(1+v_{i}\right) r_{i}, \\
& \varepsilon_{o r}=\frac{a_{o}}{3} \frac{\left(1+v_{o}\right) r_{o}}{r_{h}+r_{o}}\left[\Delta T_{o}\left(2 r_{o}+r_{h}\right)+\Delta T_{h}\left(2 r_{h}+r_{o}\right)\right],
\end{aligned}
$$

where $a_{i}$ and $a_{o}$ are the thermal expansion coefficient of inner and outer raceways, respectively, $\Delta T_{i}$ and $\Delta T_{o}$ are the temperature rise of inner and outer raceways, respectively, $v_{o}$ is Poisson's ratio of the outer raceway, $r_{i}$ and $r_{o}$ are the groove curvature radius of inner and outer raceways, respectively, and $r_{h}$ is the radius of housing.

Spindle bearings are in an O-type configuration in this paper. Substituting equations (24) and (25) into equation (29), the bearing axial expansion can be obtained, which is a function of the spindle expansion and the housing expansion:

$$
\varepsilon_{a}=\varepsilon_{h}-\varepsilon_{s} .
$$

Also, substituting equations (23), (27), and (28) into equation (30), the radial expansion of the bearing can be determined:

$$
\varepsilon_{r}=\varepsilon_{i r}-\varepsilon_{o r}-\varepsilon_{b} .
$$

\subsection{Bearing Quasi-Static Model under High Speed and} Thermal Effect. For a high-speed ball bearing, the centrifugal force and gyroscopic moment can be produced under combined axial, radial, and moment loads, which can change the contact angle between the roller and the rings and affect the load-deformation characteristics of the bearing. At the same time, the friction heat generated by balls and rings can also cause changes in the internal structure of the bearing. It is assumed that the outer ring groove curvature center is fixed in the space and the inner ring groove curvature center moves relatively to that fixed center. When the thermal expansion and the centrifugal deformation are considered, the geometrical relationship between the ball center and the inner ring and the outer ring curvature center is shown in Figure 2.

In consideration of the impact of the centrifugal deformation and the thermal expansion, the axial and radial distance between the loci of inner and outer ring groove curvature centers at any ball position can be written as follows:

$$
\begin{aligned}
& A_{1 j}=B D \sin \alpha_{0}+\delta_{a}+\theta R_{i} \cos \psi_{j}+\varepsilon_{a}, \\
& A_{2 j}=B D \cos \alpha_{0}+\delta_{r} \cos \psi_{j}+\varepsilon_{r}+\varepsilon_{c i},
\end{aligned}
$$

where $B=f_{i}+f_{o}-1, f_{i}$ and $f_{o}$ are the curvature coefficients of the inner and outer ring, respectively, $\delta_{a}$ is the relative axial displacement of the inner and outer ring, $\theta$ is the relative angular displacement, $\delta_{r}$ is the relative radial displacement of the ring centers, $\alpha_{0}$ is the original contact angular, $R_{i}$ is the radius of the locus of inner ring groove curvature centers, and $\psi_{j}$ is the azimuth of ball $j$.

Using the Pythagorean theorem, the geometric compatibility equations of the groove contact deformation are obtained [11]:

$$
\begin{array}{r}
\left(A_{1 j}-X_{1 j}\right)^{2}+\left(A_{2 j}-X_{2 j}\right)^{2}-\left[\left(f_{i}-0.5\right) D+\delta_{i j}\right]^{2}=0, \\
X_{1 j}^{2}+X_{2 j}^{2}-\left[\left(f_{o}-0.5\right) D+\delta_{o j}\right]^{2}=0,
\end{array}
$$

where $X_{1 j}$ and $X_{2 j}$ are the axial and radial projection of the distance between the ball center and the outer ring groove curvature center and $\delta_{i j}$ and $\delta_{o j}$ are the contact deformation at the inner and outer ring, respectively.

From Figure 3, according to the outer raceway control theory and the quasi-static mechanics, the equilibrium of forces of the ball $j$ in the horizontal and vertical directions is given as follows [33]:

$$
\begin{gathered}
Q_{i j} \sin \alpha_{i j}-Q_{o j} \sin \alpha_{o j}+\frac{2 M_{g j}}{D} \cos \alpha_{o j}=0, \\
Q_{i j} \cos \alpha_{i j}-Q_{o j} \cos \alpha_{o j}-\frac{2 M_{g j}}{D} \sin \alpha_{o j}+F_{c j}=0, \\
F_{c j}=\frac{1}{2} m_{b} d_{m} \omega^{2}\left(\frac{\omega_{m}}{\omega}\right)_{j}^{2} \\
M_{g j}=J\left(\frac{\omega_{r}}{\omega}\right)_{j}\left(\frac{\omega_{m}}{\omega}\right)_{j} \omega^{2} \sin \beta,
\end{gathered}
$$

where $F_{c j}$ and $M_{g j}$ are the centrifugal force and the gyroscopic moment, respectively, and $m_{b}$ is the mass of a ball.

The conditions of equilibrium of the entire bearing are established as follows:

$$
\begin{gathered}
F_{a}+F_{a 0}-\sum_{j=1}^{z} Q_{i j} \sin \alpha_{i j}=0, \\
F_{r}-\sum_{j=1}^{z} Q_{i j} \cos \alpha_{i j} \cos \psi_{j}=0, \\
M-\sum_{j=1}^{z} Q_{i j} \sin \alpha_{i j} R_{i} \cos \psi_{j}=0,
\end{gathered}
$$

where $F_{a}$ is the axial load applied to the bearing, $F_{a 0}$ is the initial preload, $F_{r}$ is the radial load of the bearing, $M$ is the moment of the bearing, and $z$ is the number of balls. $Q_{i j}=$ $K_{i j}^{\prime} \delta_{i j}^{3 / 2}$ and $Q_{o j}=K_{o j}^{\prime} \delta_{o j}^{3 / 2}$, where $K_{i j}^{\prime}$ is the load-deflection constant between the rolling element and the inner ring and $K_{o j}^{\prime}$ is the load-deflection constant between the rolling element and the outer ring. 


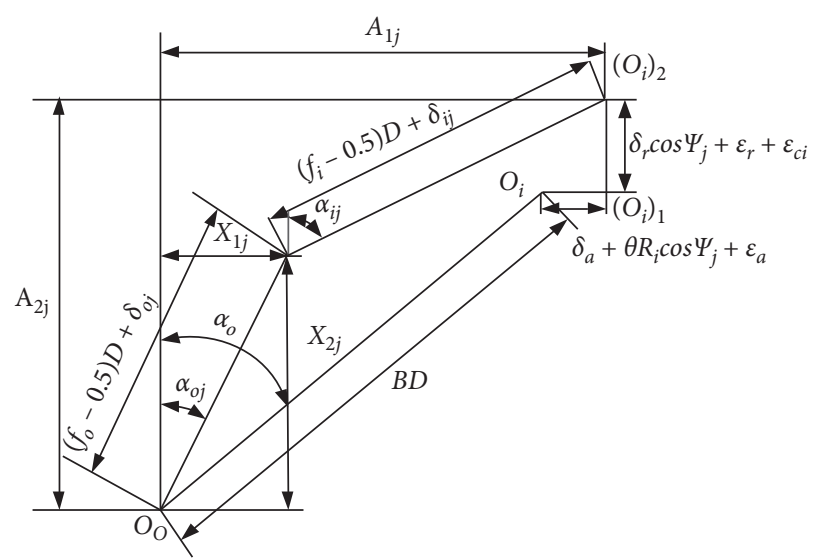

FIgURe 2: Positions of ball center and ring groove curvature centers.

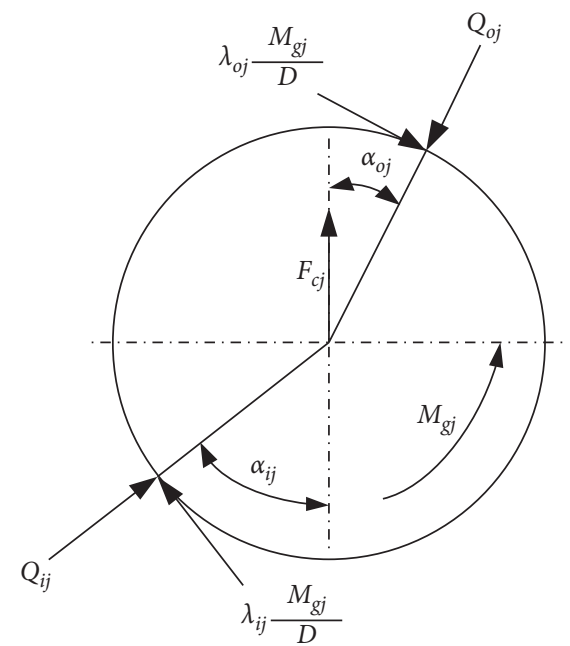

FIGURE 3: Mechanical analysis of the rolling element.

Using the Hertz contact theory, the relationship between normal load and normal deformation of the rolling element is defined as follows [34]:

$$
\delta=\Gamma\left[\frac{9 \sum \rho}{2 \pi k E^{\prime 2} \Sigma}\right]^{1 / 3} Q^{2 / 3} .
$$

Differentiating equation (42), the Hertz contact stiffness is

$$
\begin{gathered}
K_{c}=1.5\left[\frac{\pi k E^{\prime}}{3 \Gamma}\right]^{2 / 3}\left[\frac{2 \sum}{\Gamma \sum \rho}\right]^{1 / 3} Q^{1 / 3}, \\
k=1.0339\left[\frac{\sum \rho_{\mathrm{II}}}{\sum \rho_{\mathrm{I}}}\right]^{0.636}, \\
\Gamma=1.5277+0.6023 \ln \left[\frac{\sum \rho_{\mathrm{II}}}{\sum \rho_{\mathrm{I}}}\right], \\
\sum=1.0003+0.5968 \frac{\sum \rho_{\mathrm{I}}}{\sum \rho_{\mathrm{II}}}
\end{gathered}
$$

where $Q$ is the contact load and $\sum \rho_{\text {I }}$ and $\sum \rho_{\text {II }}$ are the sum of curvature of the contact point in the I and II plane.

The contact load and contact angle between each ball and raceways may be determined by equations (31)-(41). Substituting these quantities into equation (43), the contact stiffness is obtained.

\section{Comprehensive Stiffness Model of the Bearing}

For low-speed rolling bearings, the contact stiffness can be approximately expressed as the bearing overall stiffness. However, when the angular contact ball bearings operate at high speeds, the magnitude of the film stiffness is close to that of the contact stiffness under the combined influence of the high speed and the film lubrication. Therefore, the contact stiffness and film stiffness are connected in series to represent the comprehensive stiffness. At the same time, different contact angles of the inner and outer ring result in different stiffness of the inner and outer ring. The comprehensive stiffness between the ball $j$ and the ring is expressed as follows [35]:

$$
\frac{1}{K_{j}}=\frac{1}{K_{c j}}+\frac{1}{K_{o i l j}},
$$

where $K_{c j}$ is the contact stiffness between the ball $j$ and the ring and $K_{o i l j}$ is the film stiffness between the ball $j$ and the ring.

According to Harris' computational formula of stiffness, the axial stiffness and the radial stiffness between the ball $j$ and the ring can be written as follows [11]:

$$
\begin{aligned}
& K_{a j}=K_{j} \sin ^{2} \alpha_{j}, \\
& K_{r j}=K_{j} \cos ^{2} \alpha_{j},
\end{aligned}
$$

where $\alpha_{j}$ is the contact angular between the ball $j$ and the ring.

Equations (45) and (46) are then used to determine the comprehensive bearing radial stiffness, axial stiffness, and angle stiffness, which are calculated as 


$$
\begin{aligned}
K_{r} & =\sum_{j=1}^{z} \frac{K_{r i j} K_{r o j}}{K_{r i j}+K_{r o j}} \cos ^{2} \psi_{j}, \\
K_{a} & =\sum_{j=1}^{z} \frac{K_{a i j} K_{a o j}}{K_{a i j}+K_{a o j}}, \\
K_{\theta} & =\frac{d_{m}^{2}}{4} \sum_{j=1}^{z} \frac{K_{a i j} K_{a o j}}{K_{a i j}+K_{a o j}} \cos ^{2} \psi_{j},
\end{aligned}
$$

where $K_{a i j}$ and $K_{r i j}$ are the axial stiffness and radial stiffness between the ball $j$ and the inner ring and $K_{a o j}$ and $K_{r o j}$ are the axial stiffness and radial stiffness between the ball $j$ and the outer ring.

\section{Example of the Integrated Bearing Stiffness Calculation}

5.1. Numerical Methods. The integrated model for the comprehensive bearing stiffness is solved by the flowchart given in Figure 4 . When the bearing runs at high speed, firstly, the centrifugal expansion of the inner ring was calculated. Secondly, the thermal expansion of the bearing components was obtained according to the temperature rise. Thirdly, the geometric relationships of Jones' bearing model were modified according to various deformations. The trust region method [36] and Levenberg-Marquardt algorithm [37] were adopted to solve the quasi-static modified model of the bearing. The model was resolved by using the calculated deformation to revise the internal geometrical relationship of the bearing. The above steps were repeated until the solution of the equations met the convergence precision and then the contact stiffness of the bearing was obtained. Moreover, the film stiffness considering spinning was calculated based on the equations of the spin angular velocity and the elastohydrodynamic lubrication (EHL) basic equations. Finally, the comprehensive stiffness was derived from combining the contact stiffness and the film stiffness.

\subsection{Numerical Results and Discussion}

5.2.1. Validation of the Present Model. In order to verify the accuracy of the updated mathematical model of the angular contact ball bearing, the results of an example is illustrated to compare the results with [23]. The values of bearing parameters are listed in Table 1. Moreover, the oil temperature is $300 \mathrm{~K}$. The original dynamic viscosity is $0.027 \mathrm{~Pa} \cdot \mathrm{s}$. The pressure-viscosity coefficient is $2.3 \times 10^{-8} \mathrm{~Pa}^{-1}$. The specific heat of oil is $2000 \mathrm{~J} /(\mathrm{kg} \cdot \mathrm{K})$. The thermal conductivity of oil is $0.14 \mathrm{~W}$. The results are compared in Figure 5.

As illustrated in Figure 5, there is only slight difference between two sets of the contact stiffness curves and the maximum error is about $4 \%$. It is obvious that when the speed increases, the bearing stiffness of both models decrease. From Figure 5, it can also be found that this conclusion is basically consistent with the trend of the existing dynamic stiffness.

5.2.2. Effect of the Bearing Speed on the Film Stiffness. In Figure 6, it can be seen that as speed increases, the film stiffness of inner and outer raceways decrease. The reason for this phenomenon is that the lubricating oil flow rate increases with the increase in speed, and the film thickness increases, but the load does not change much. The change trend of the film stiffness was observed in [26]. The results are consistent with this calculation results.

\subsubsection{Effect of Both the Bearing Centrifugal Deformation and} the Thermal Deformation on the Contact Stiffness. Figure 7 shows the effect of the ring centrifugal and thermal deformation on the contact stiffness of the bearing. It can be seen that the radial, axial, and angular stiffness decrease nonlinearly with the increase in speed, such phenomena are due to the fact that as the rotation speed increases, the gyroscopic moment and centrifugal force increase and the centrifugal force causes the contact angle of the inner ring to increase and that of the outer ring to decrease, which softens the bearing stiffness. This situation was observed in experiments carried out by Wang et al. [38]. It is also found that the ring centrifugal deformation and the thermal deformation have more impact on the radial stiffness than on the axial and angle stiffness at high speed. In Figures $7(\mathrm{a})-7(\mathrm{c})$, the maximum and minimum percentage difference between the results are $50 \%$ and $7 \%, 20 \%$ and $4 \%$, and $15 \%$ and $3 \%$, respectively. That is because if the effect of deformation is considered, the radial dynamic load of the rolling bearing increases with the speed; meanwhile, the deformation of the ring becomes larger. However, the increase in the radial deformation is overshadowed by the increase in the load, which results in an increase in radial dynamic stiffness.

\subsubsection{Effect of Both the Bearing Deformation and the EHL on} the Comprehensive Stiffness. Figure 8 shows the influence of EHL, the inner ring centrifugal deformation, and the thermal deformation on the comprehensive stiffness of the rolling bearing. When EHL effect is considered, all of the comprehensive radial, axial, and angular stiffness are lower without consideration of EHL effect. In Figures 8(a)-8(c), the maximum and minimum percentage difference between the results are $35 \%$ and $1 \%, 18 \%$ and $5 \%$, and $18 \%$ and $5 \%$, respectively. To the bearing radial stiffness, when the speed increases, the thermal deformation and the centrifugal deformation can enhance bearing contact stiffness, while the film stiffness decreases. Thus, the magnitude of the contact stiffness is close to that of the film stiffness. They are combined together through the series connection, which leads to a sharp decrease in the comprehensive stiffness. However, the centrifugal deformation and thermal deformation at high speed have little effect on the axial and angular stiffness, and there is little change in contact 


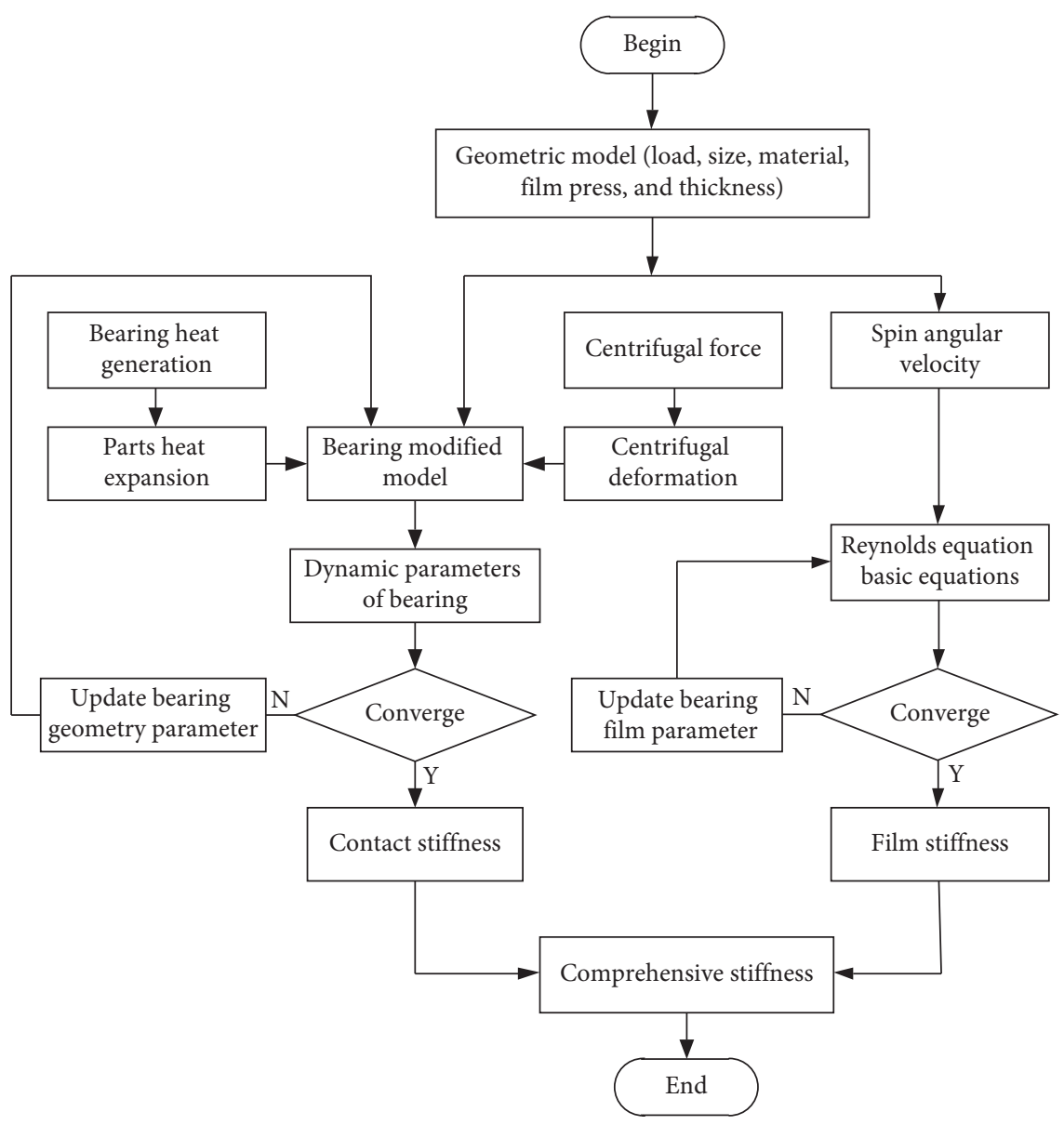

Figure 4: Flowchart for the present method.

TABle 1: Parameters of angular contact ball bearing.

\begin{tabular}{lc}
\hline Parameter & Value \\
\hline Inner ring diameter $(\mathrm{mm})$ & 65 \\
Outer ring diameter $(\mathrm{mm})$ & 100 \\
Ball diameter $(\mathrm{mm})$ & 11.11 \\
Number of balls & 20 \\
Original contact angle $\left(^{\circ}\right)$ & 15 \\
Inner ring groove radius $(\mathrm{mm})$ & 5.9994 \\
Outer ring groove radius $(\mathrm{mm})$ & 6.3327 \\
\hline
\end{tabular}

stiffness. The axial and angular stiffness are affected by the EHL, and the film stiffness plays a major role; therefore, the change in the axial and angular stiffness is not obvious with the speed.

Figure 9 illustrates the comparison between the calculation results in this paper and in [23]. It can be seen that the comprehensive bearing stiffness considering the spinning is larger than that without consideration of the spinning. The reason for this phenomenon is that the film stiffness considering spin motion increases, which leads to the increase in the integrated stiffness. The maximum percentage difference between the results is $6 \%$. As a whole, the spinning motion has great influence on the film

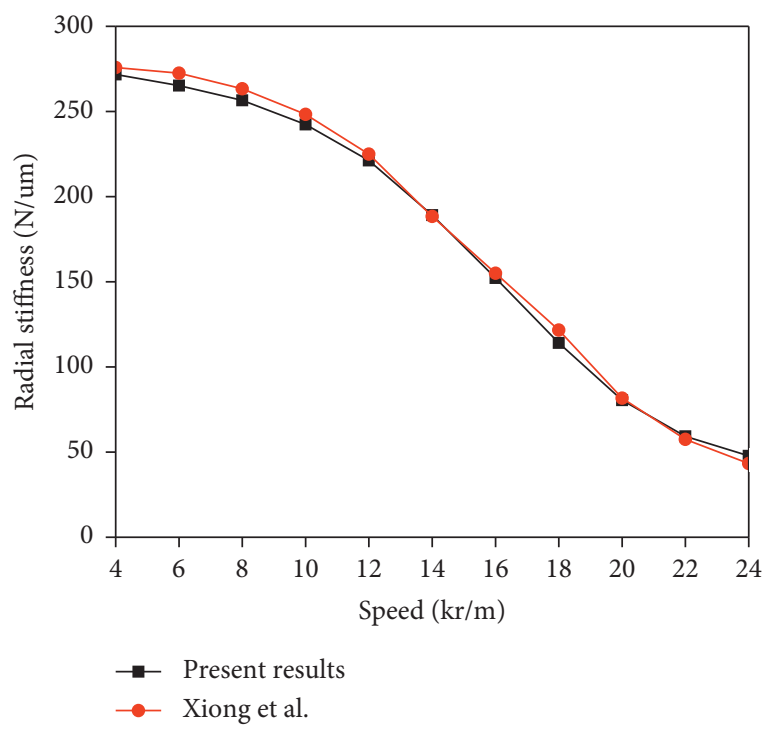

Figure 5: Comparison of two different models.

stiffness, which was already observed in previous work of the authors [26], but it has little influence on the comprehensive stiffness. The reason for this is the film stiffness 


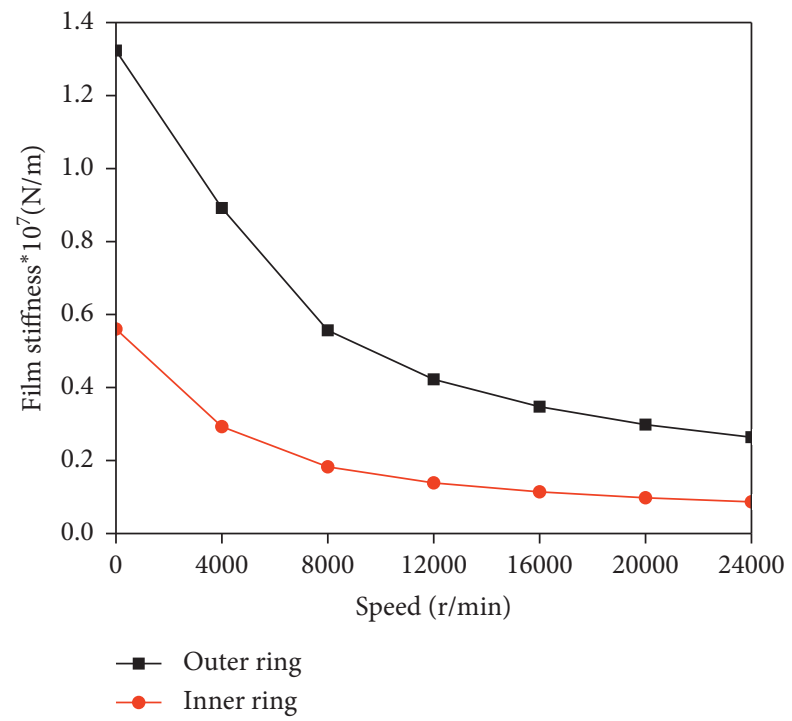

FIGURE 6: Relationship between the film stiffness and the speed.

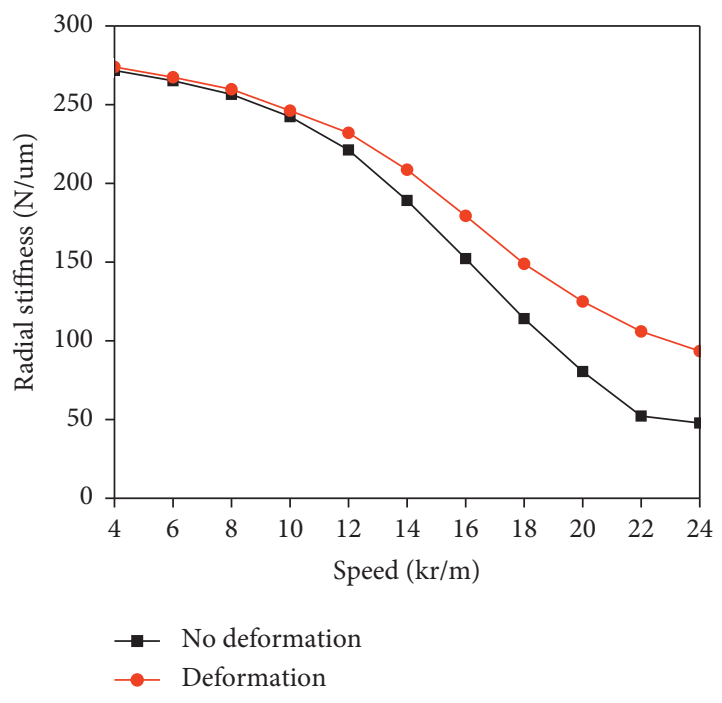

(a)

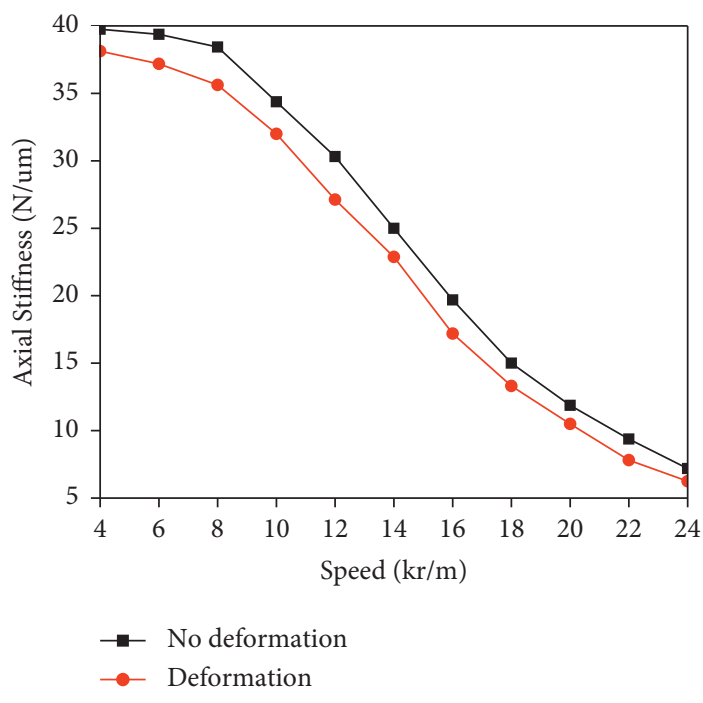

(b)

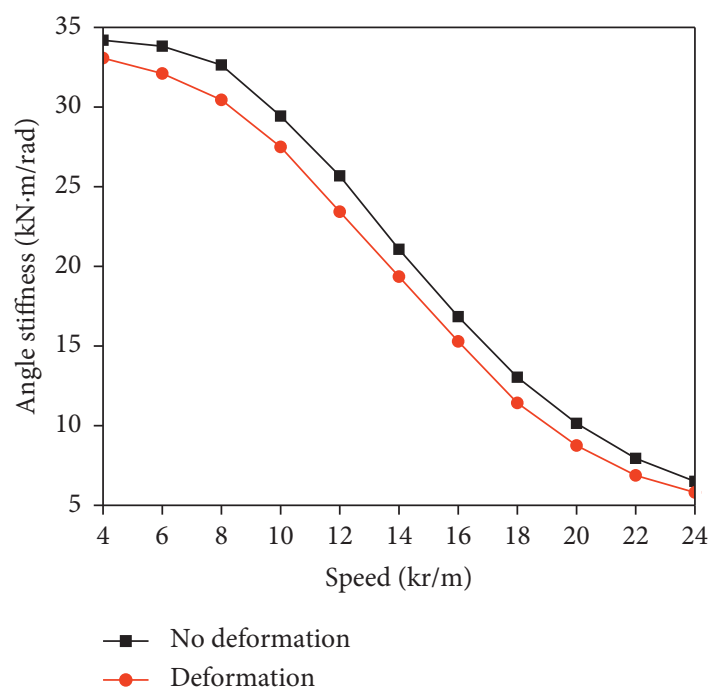

(c)

FiguRE 7: Bearing contact stiffness at different speeds. (a) Radial contact stiffness, (b) axial contact stiffness, and (c) angular contact stiffness. 


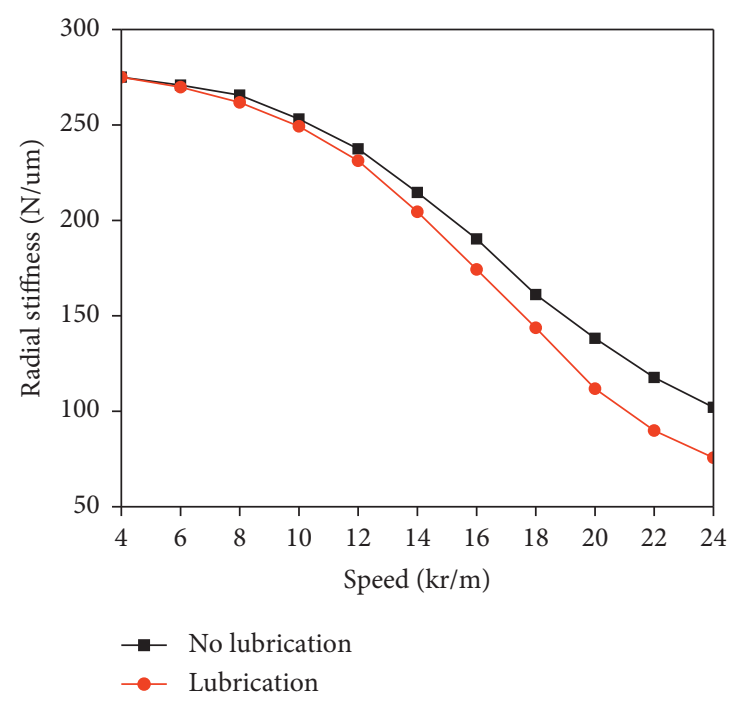

(a)

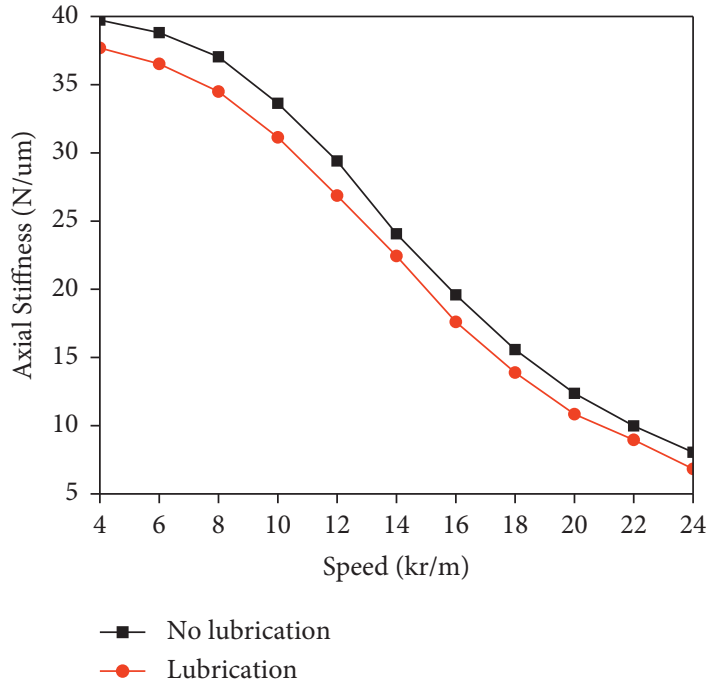

(b)

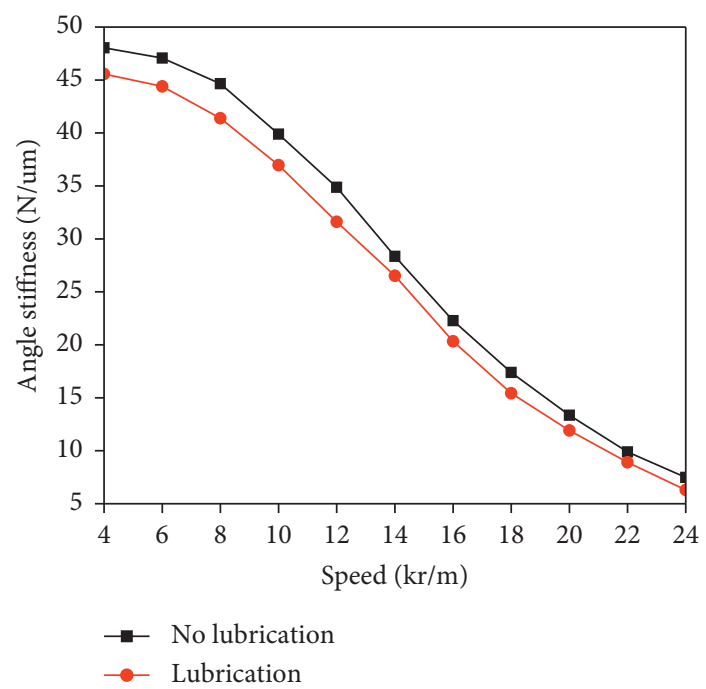

(c)

FIGURE 8: Comprehensive bearing stiffness at different speeds. (a) Radial comprehensive stiffness, (b) axial comprehensive stiffness, and (c) angular comprehensive stiffness.

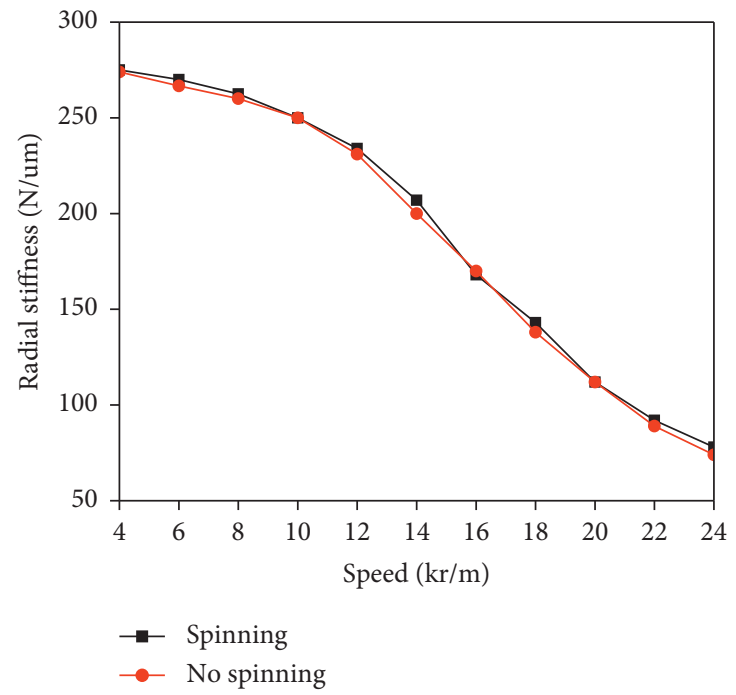

FIGURE 9: Effect of spin motion on the comprehensive stiffness. 
is combined with the contact stiffness by the series connection, which weakens the spinning effect.

\section{Conclusions}

A modified calculation model of comprehensive stiffness of the angular contact ball bearing is proposed in this paper. The model takes into account the centrifugal deformation of the inner ring and the thermal deformation of the bearing, as well as the spinning motion of the ball. The change laws and influencing factors of integrated dynamic stiffness of the bearing at high speed are investigated systematically. The following conclusions can be drawn:

(1) At high speed, the centrifugal expansion and thermal expansion of bearing components increase with the increase in speed, so the influence of speed must be taken into account when the dynamic stiffness of bearing is calculated.

(2) When the centrifugal effect and thermal effect on the stiffness are considered, the increase in speed can cause the radial stiffness to increase; however, the increase in speed can cause the axial and angular stiffness to decrease.

(3) When the influence of deformations and the EHL on the stiffness is taken into account, the comprehensive stiffness of the bearing decreases. The decrease of the radial stiffness is greater than that of the axial and angular stiffness.

(4) In consideration of the effects of the spin motion of the ball, the dynamic stiffness of the bearing increases, whereas the variation range is small.

(5) It is well known that the wear would have a great influence on the dynamic stiffness at high speed. Thus, the effect of the wear on the stiffness will be studied in our future work.

\section{Data Availability}

The data used to support the findings of this study are included within the supplementary information file.

\section{Conflicts of Interest}

The authors declare that they have no conflicts of interest.

\section{Acknowledgments}

This research was financially supported by the National Science Foundation of China (grant no. 51465035) and the State Scholarship Fund (file no. 201608625027).

\section{Supplementary Materials}

Value data-4951828. (Supplementary Materials)

\section{References}

[1] Y. F. Zhang, X. H. Li, J. Hong et al., "Investigation of multiple spindle characteristics under non-uniform bearing preload,"
Advanced in Mechanical Engineering, vol. 8, no. 2, pp. 1-12, 2016.

[2] E. Abele, Y. Altintas, and C. Brecher, "Machine tool spindle units," CIRP Annals, vol. 59, no. 2, pp. 781-802, 2010.

[3] J. Liu, Z. Xu, L. Zhou, W. Yu, and Y. Shao, "A statistical feature investigation of the spalling propagation assessment for a ball bearing," Mechanism and Machine Theory, vol. 131, pp. 336-350, 2019.

[4] Y. Qin, F. Cao, Y. Wang, W. Chen, and H. Chen, "Dynamics modelling for deep groove ball bearings with local faults based on coupled and segmented displacement excitation," Journal of Sound and Vibration, vol. 447, pp. 1-19, 2019.

[5] J. Liu, "A dynamic modelling method of a rotor-roller bearing-housing system with a localized fault including the additional excitation zone," Journal of Sound and Vibration, vol. 469, Article ID 115144, 2020.

[6] T. Hu, G. F. Yin, and C. Y. Deng, "Integrated thermo-mechanical model and analysis of angular contact ball bearing," Journal of Sichuan University (Engineering Science Edition), vol. 46, no. 4, pp. 189-198, 2014.

[7] Y. Altintas and Y. Cao, "Virtual design and optimization of machine tool spindles," CIRP Annals, vol. 54, no. 1, pp. 379-382, 2005.

[8] J. Kraus, J. J. Blech, and S. G. Braun, "In situ determination of rolling bearing stiffness and damping by modal analysis," Journal of Vibration and Acoustics, vol. 109, no. 3, pp. 235240, 1987.

[9] A. B. Jones, "Ball motion and sliding friction in ball bearings," Journal of Basic Engineering, vol. 81, no. 1, pp. 1-12, 1959.

[10] T. A. Harris and M. H. Mindel, "Rolling element bearing dynamics," Wear, vol. 23, no. 3, pp. 311-337, 1973.

[11] T. A. Harris, Rolling Bearing Analysis, John Wiley \& Sons, New York, USA, 1984.

[12] P. K. Gupta, Advanced Dynamics of Rolling Elements, Springer-Verlag, New York, USA, 1984.

[13] C.-W. Lin, J. F. Tu, and J. Kamman, "An integrated thermomechanical-dynamic model to characterize motorized machine tool spindles during very high speed rotation," International Journal of Machine Tools and Manufacture, vol. 43, no. 10, pp. 1035-1050, 2003.

[14] H. Cao, T. Holkup, and Y. Altintas, "A comparative study on the dynamics of high speed spindles with respect to different preload mechanisms," The International Journal of Advanced Manufacturing Technology, vol. 57, no. 9-12, pp. 871-883, 2011.

[15] F. M. A. El-Saeidy and M. A. Fawzi, "Time-varying total stiffness matrix of a rigid machine spindle-angular contact ball bearings assembly: theory and analytical/experimental verifications," Shock and Vibration, vol. 18, no. 5, pp. 641-670, 2011.

[16] A. Gunduz and R. Singh, "Stiffness matrix formulation for double row angular contact ball bearings: analytical development and validation," Journal of Sound and Vibration, vol. 332, no. 22, pp. 5898-5916, 2013.

[17] D. Noel, M. Ritou, and B. Furet, "Complete analytical expression of the stiffness matrix of angular contact ball bearing," Journal of Sound and Vibration, vol. 135, no. 135, pp. 1-8, 2013.

[18] Y. Cao and Y. Altintas, "A general method for the modeling of spindle-bearing systems," Journal of Mechanical Design, vol. 126, no. 6, pp. 1089-1104, 2004.

[19] B. Fang, L. Zhang, X. T. Qu et al., “Theoretical and experimental research of angular contact ball bearing," Journal of 
Jilin University (Engineering and Technology Edition), vol. 42, no. 4, pp. 840-844, 2012.

[20] G. D. Hagiu and M. D. Gafitanu, "Dynamic characteristics of high speed angular contact ball bearings," Wear, vol. 211, no. 1, pp. 22-29, 1997.

[21] K. Guo, S. H. Yuan, Y. Y. Zhang et al., "Study on the calculation method of ball bearing mechanical characteristics considering elastohydrodynamic lubrication with spinning," Journal of Mechanical Engineering, vol. 49, no. 15, pp. 62-67, 2013.

[22] Y. Guo and R. G. Parker, "Stiffness matrix calculation of rolling element bearings using a finite element/contact mechanics model," Mechanism and Machine Theory, vol. 51, pp. 32-45, 2012.

[23] W. L. Xiong, Z. S. Zhao, Y. Zhou et al., "Research on dynamic stiffness of ball bearing considering ferrule deformation and elastohydrodynamic lubrication," Chinese Journal of $\mathrm{Me}$ chanical Engineering, vol. 26, no. 11, pp. 1421-1428, 2015.

[24] J. Liu, C. K. Tang, H. Wu, Z. Xu, and L. Wang, "An analytical calculation method of the load distribution and stiffness of an angular contact ball bearing," Mechanism and Machine Theory, vol. 142, Article ID 103597, 2019.

[25] C. Ding, F. Z. Zhou, J. Zhu et al., "Raceway control assumption and the determination of rolling element attitude angle," Chinese Journal of Mechanical Engineering, vol. 37, no. 2, pp. 58-61, 2001.

[26] M. X. Wu, W. Wu, J. B. Hu et al., "Oil film stiffness calculation of high speed angular contact ball bearing considering spinning," Journal of Vibration and Shock, vol. 33, no. 10, pp. 38-42, 2014.

[27] C. J. A. Roelands, Correlational Aspects of the ViscosityTemperature-Pressure Relationship of Lubricating Oils, Druk U R B, Groningen, Netherlands, 1966.

[28] S. Z. Wen and P. R. Yang, Elastohydrodynamic Lubrication, Tsinghua University Press, Beijing, China, 1992.

[29] J. L. Tian, J. Hong, Y. S. Zhu et al., "Thermo-mechanical coupling model and dynamical characteristics of machining spindle-bearing system," Journal of Xi'an Jiaotong University, vol. 46, no. 7, pp. 63-68, 2012.

[30] F. H. Li, Dynamic Stiffness Analysis of Rolling Bearing Based on Multi-Parameter Coupling, Lanzhou University of Technology, Lanzhou, China, 2017.

[31] X. J. Jiang, J. J. Ma, and L. C. Zhao, “Analysis on heat displacement of components of high speed precision angular contact ball bearing," Bearing, vol. 1, pp. 5-7, 2001.

[32] B. R. Jorgensen and Y. C. Shin, "Dynamics of machine tool spindle/bearing systems under thermal growth," Journal of Tribology, vol. 119, no. 4, pp. 875-882, 1997.

[33] Y. Qin, C. Li, F. Cao, and H. Chen, "A fault dynamic model of high-speed angular contact ball bearings," Mechanism and Machine Theory, vol. 143, p. 103627, 2020.

[34] B. J. Hamrock and D. Dowson, Ball Bearing Lubrication, China Machine Press, Beijing, China, 1988, in Chinese.

[35] Y. J. Yu, G. D. Chen, and J. S. Li, "Research on stiffness characteristics of high speed angular-contact ball bearing considering elastohy-drodynamic lubrication effect," Journal of North-Western Polytechnical University, vol. 34, no. 1, pp. 125-131, 2016.

[36] C. Li and Q. Pan, "Adaptive optimization methodology based on Kriging modeling and a trust region method," Chinese Journal of Aeronautics, vol. 32, no. 2, pp. 281-295, 2019.

[37] S.-A. Blaifi, S. Moulahoum, B. Taghezouit, and A. Saim, “An enhanced dynamic modeling of PV module using Levenberg-
Marquardt algorithm," Renewable Energy, vol. 135, pp. 745760, 2019.

[38] X. Wang, Y. Guo, and T. Chen, "Measurement research of motorized spindle dynamic stiffness under high speed rotating," Shock and Vibration, vol. 2015, Article ID 284126, 11 pages, 2015. 